\title{
Severe combined immunodeficiency caused by deficiency in either the $\delta$ or the $\varepsilon$ subunit of CD3
}

\author{
Geneviève de Saint Basile, ${ }^{1}$ Frédéric Geissmann, ${ }^{2}$ Elisabeth Flori, ${ }^{3}$ \\ Béatrice Uring-Lambert, ${ }^{4}$ Claire Soudais, ${ }^{1}$ Marina Cavazzana-Calvo, ${ }^{1}$ \\ Anne Durandy, ${ }^{1}$ Nada Jabado, ${ }^{5}$ Alain Fischer, ${ }^{1,5}$ and Françoise Le Deist ${ }^{1,6}$

\begin{abstract}
1Unité Développement Normal et Pathologique du Système Immunitaire, INSERM U 429 and 'Laboratoire d'Anatomie Pathologique, Hôpital Necker-Enfants Malades, Paris, France. ${ }^{3}$ Service de Cytogénétique and ${ }^{4}$ Laboratoire d'Immunologie et d'Hématologie, Hôpitaux Universitaires de Strasbourg, Strasbourg, France. 5Unité d'Immunologie et d'Hématologie Pédiatrique and
\end{abstract} \\ ${ }^{6}$ Centre d'Étude des Déficits Immunitaires, Hôpital Necker-Enfants Malades, Paris, France.
}

\begin{abstract}
We investigated the molecular mechanism underlying a severe combined immunodeficiency characterized by the selective and complete absence of T cells. The condition was found in 5 patients and 2 fetuses from 3 consanguineous families. Linkage analysis performed on the 3 families revealed that the patients were carrying homozygous haplotypes within the 11q23 region, in which the genes encoding the $\gamma, \delta$, and $\varepsilon$ subunits of CD3 are located. Patients and affected fetuses from 2 families were homozygous for a mutation in the CD3D gene, and patients from the third family were homozygous for a mutation in the CD3E gene. The thymus from a CD3 $\delta$ deficient fetus was analyzed and revealed that $T$ cell differentiation was blocked at entry into the double positive $\left(\mathrm{CD}^{+} \mathrm{CD8}^{+}\right)$stage with the accumulation of intermediate $\mathrm{CD} 4-$ single positive cells. This indicates that $\mathrm{CD} 3 \delta$ plays an essential role in promoting progression of early thymocytes toward double-positive stage. Altogether, these findings extend the known molecular mechanisms underlying severe combined immunodeficiency to a new deficiency, i.e., CD3 $\varepsilon$ deficiency, and emphasize the essential roles played by the CD3 $\varepsilon$ and CD3 $\delta$ subunits in human thymocyte development, since these subunits associate with both the pre-TCR and the TCR.
\end{abstract}

\section{Introduction}

Severe combined immunodeficiencies (SCIDs) are a group of genetic disorders characterized by profoundly defective $T$ cell differentiation (1). Several SCID conditions have been defined on the basis of enzymatic, genetic, and immunological criteria (2). Adenosine deaminase deficiency results in a profound impairment of T, B, and NK lymphocyte development (3). One subset of SCIDs is characterized by the arrest of both $\mathrm{T}$ and $\mathrm{B}$ lymphocyte differentiation ( $\left.\mathrm{T}^{-} \mathrm{B}^{-} \mathrm{SCID}\right)$, whereas in a different subset $\mathrm{B}$ cell differentiation is unaffected but $\mathrm{NK}$ cell differentiation is impaired ( $\left.\mathrm{T}^{-} \mathrm{B}^{+} \mathrm{NK}^{-} \mathrm{SCID}\right)$. The molecular bases of many of these conditions are now known and involve defects in either $\mathrm{T}$ and $\mathrm{B}$ cell receptor $\mathrm{V}(\mathrm{D}) \mathrm{J}$ recombination factors, i.e., Rag-1, Rag-2, or Artemis, in $\mathrm{T}^{-} \mathrm{B}^{-} \mathrm{SCID}(4,5)$, or IL-dependent T cell and NK cell differentiation due to mutation in the IL $2 R G$ gene ( $\gamma$ c deficiency) or the $\gamma c$-associated JAK3 kinase gene (Jak-3 deficiency) in $\mathrm{T}^{-} \mathrm{B}^{+} \mathrm{NK}^{-} \mathrm{SCID}$ $(6,7)$. In addition, another SCID subset is characterized by an absence of mature $\mathrm{T}$ lymphocytes in the presence of normally proceeding maturation of $\mathrm{NK}$ and $\mathrm{B}$ cells ( $\left.\mathrm{T}^{-} \mathrm{B}^{+} \mathrm{NK}^{+} \mathrm{SCID}\right)$. Deficiency of the $\alpha$ chain of the IL-7 receptor (IL-7R $\alpha$ ) (8) is the predominant form of $\mathrm{T}^{-} \mathrm{B}^{+} \mathrm{NK}^{+}$ SCID, but 2 cases associated with CD 45 deficiency have been reported $(9,10)$. More recently, a mutation in the $C D 3 D$ gene was identified in 3 members of a kindred with the $\mathrm{T}^{-} \mathrm{B}^{+} \mathrm{NK}^{+}$form of SCID (11).

Intrathymic $\mathrm{T}$ lymphocyte differentiation requires appropriate signals from the pre-TCR (PreT $\alpha /$ TCR $\beta)$ and/or the TCR (TCR $\alpha / \beta$ or TCR $\gamma / \delta)(12-14)$. The pre-TCR, TCR $\alpha / \beta$, and TCR $\gamma / \delta$ are associated with 3 signal-transducing complexes, namely the CD3 $\delta \varepsilon$ and $\mathrm{CD} 3 \gamma \varepsilon$ heterodimers and a disulfide-linked $\operatorname{CD} 3 \xi \zeta(15,16)$. Howev-

Nonstandard abbreviations used: BMT, bone marrow transplantation; IL-7R $\alpha$, $\alpha$ chain of the IL-7 receptor; ISP, intermediate single positive; P, patient (PI, PII, etc.). Conflict of interest: The authors have declared that no conflict of interest exists.

Citation for this article: J. Clin. Invest. 114:1512-1517 (2004).

doi:10.1172/JCI200422588 er, in humans, the respective roles of the different $\mathrm{CD} 3$ chains in $\mathrm{T}$ cell differentiation are not well known. In $\mathrm{CD} 3 \gamma^{-/-}$mice, thymocyte differentiation is blocked at the double negative stage (17), which suggests that the $\gamma \varepsilon$ dimer is essential at an earlier intrathymic maturation step than the $\delta \varepsilon$ dimer. In these mice, $\mathrm{T}$ cell differentiation is probably blocked because the pre-TCR is unable to transduce the signal for progression. In contrast, thymocytes from $\mathrm{CD} 3 \delta^{-/-}$mice do progress to the double positive stage (18).

Studies of human $\mathrm{T}$ cell immunodeficiencies associated with a defect in any one of these three subunits may provide insights into both the redundant and the nonredundant roles of the CD3 molecules. Until recently, only 2 immunodeficiencies associated with a CD3 chain defect had been described. CD3 $\gamma$ deficiency did not impair intrathymic $\mathrm{T}$ cell differentiation. In these cases, peripheral CD4 T cells and the few CD8 T cells present expressed a CD3 $\gamma$-deficient CD 3 complex $(19,20)$. Conversely, the study of a T- SCID condition associated with a CD3 $\delta$ deficiency suggested that the CD $3 \delta$ subunit is essential for $\mathrm{T}$ cell development in humans (11). In this study we describe a $\mathrm{T}^{-} \mathrm{B}^{+} \mathrm{NK}^{+} \mathrm{SCID}$ phenotype associated with either a CD3 $\delta$ deficiency found in 2 families - with a hitherto undescribed mutation in 1 of the families - or a CD $3 \varepsilon$ deficiency in 1 family.

\section{Results}

Immunological characteristics. No $\mathrm{CD}^{+} \mathrm{T}$ lymphocytes were detected in any of the patients tested or in fetal blood samples from family III (Table 1). B lymphocytes were detected in all tested cases. Counts were at the upper limit of the normal range. CD56 $6^{+} \mathrm{NK}$ cells were detected in the blood of the patient III-2 (PIII-2) and PIII-3 fetuses and in both patients tested (Table 1). NK cell counts were either within the normal range (PI-3) or high (PII-2). Serum IgG levels after 4 months of age, when they could be evaluated, were low (PII-1 and PIII-1). IgA was not detected in the serum of any of the patients. In contrast, $\operatorname{IgM}$ was detected in all patients 


\section{Table 1}

Patients' immunological investigations

\begin{tabular}{|c|c|c|c|c|c|c|c|c|}
\hline Family & \multicolumn{2}{|r|}{ I } & \multicolumn{2}{|c|}{ II } & \multicolumn{3}{|c|}{ III } & \multirow{3}{*}{$\begin{array}{c}\text { Age-matched } \\
\text { control } \\
\text { values }\end{array}$} \\
\hline Consanguinity & & + & & + & & + & & \\
\hline Patients (P) & PI-2 & PI-3 & PII-1 & PII-2 & PIII-1 & PIII-2 & PIII-3 & \\
\hline $\begin{array}{l}\text { Age at first } \\
\text { manifestation (mo) }\end{array}$ & 1 & - & 3 & - & 2 & $\begin{array}{l}\text { Fetus } \\
23 w k\end{array}$ & $\begin{array}{l}\text { Fetus } \\
21 w k\end{array}$ & \\
\hline Age at investigation (mo) & 2 & 1.5 & 5 & 0.1 & 5 & & & \\
\hline Lymphocytes/ul & 1,105 & 2.500 & 2,290 & 3.050 & 1.980 & 592 & 1,920 & $\begin{array}{c}1,000-1,500 \\
2,700-13,500\end{array}$ \\
\hline $\mathrm{CD} 3 / \mu \mathrm{l}(\%)$ & ND & $0(0)$ & ND & $<30(<1)$ & $0(0)$ & $11(2)$ & $90(5)$ & $\begin{array}{c}650-1,100(65-75) \\
2,100-12,000(56-87)\end{array}$ \\
\hline $\mathrm{CD} 4 / \mu \mathrm{l}(\%)$ & ND & $<25(<1)$ & ND & $<30(<1)$ & $<19(<1)$ & $<8(<1)$ & $<20(<1)$ & $\begin{array}{c}500-950(50-65) \\
1,500-8,100(33-66)\end{array}$ \\
\hline CD8/ul (\%) & ND & 6 & ND & 17 & 39 & $9(1.5)$ & $28(1.5)$ & $\begin{array}{c}100-225(8-15) \\
450-4,000\end{array}$ \\
\hline CD19/ul (\%) & ND & $1,750(70)$ & $1,489(65$ & ${ }^{A} 1,677(55)$ & $1,782(90)$ & $415(56)$ & $1,670(87)$ & $\begin{array}{l}350-525(23-32) \\
700-1,500(5-29)\end{array}$ \\
\hline CD56/ul (\%) & ND & $300(12)$ & ND & $1,342(44)$ & ND & $55(6.9)^{\mathrm{B}}$ & $77(4)^{\mathrm{B}}$ & $\begin{array}{c}20-55(1-3) \\
300-700(2-15)\end{array}$ \\
\hline $\operatorname{lgG~mg/dl~}$ & 360 & 498 & 170 & NE & 160 & NE & NE & $270-530$ \\
\hline $\lg A \mathrm{mg} / \mathrm{dl}$ & $<4$ & $<4$ & $<1$ & NE & $<4$ & NE & NE & $2-22$ \\
\hline lgM mg/dl & 20 & 42 & $55-117$ & NE & 25 & NE & NE & $\begin{array}{l}36-56 \\
40-84\end{array}$ \\
\hline
\end{tabular}

${ }^{A B}$ cells were quantified as HLA class II-positive lymphocytes. ${ }^{B}$ NK cells were quantified as CD2+CD3- lymphocytes. ND, not done; NE, could not be evaluated.

and was below or within the normal range (Table 1). Serum IgM levels did not differ from those found in the samples from patients with $\mathrm{B}^{+}$SCID caused by $\gamma c$ or JAK3 deficiency.

Identification of mutations in the CD3D or CD $3 E$ genes. Segregation analysis of polymorphic markers spanning the 19p13.1 (JAK3) and $5 \mathrm{p} 13(I L 7 R)$ chromosomal regions revealed that the patients from the 3 families carried heterozygous haplotypes in these 2 loci (data not shown). Thus, the $\mathrm{T}^{-} \mathrm{B}^{+} \mathrm{NK}^{+} \mathrm{SCID}$ phenotypes of the 3 families did not result from mutations in the JAK3 and $I L 7 R$ genes. In contrast, all patients from the 3 families displayed a homozygous haplotype segregation of polymorphic markers for 11q23 (Figure 1). The CD3 locus has been mapped to this chromosomal region, and therefore these data strongly suggest that the $\mathrm{T}^{-} \mathrm{B}^{+} \mathrm{NK}^{+}$SCID phenotypes are a result of a defect in one of the $\mathrm{CD} 3$ subunits. A search for a mutation in the gene encoding the $C D 3 D$ chain was carried out on genomic DNA from the patients. A homozygous C279A substitution, which results in a C93X nonsense codon, was identified in CD3D exon 3 in PII-2 (Figure 2). A homozygous C202T substitution, which results in a R68X nonsense codon, was found in $C D 3 D$ exon 2 in PIII-3 (Figure 2). Both normal and mutated CD $3 D$ alleles were detected in the genomic DNA of the parents in the 2 families (as well as in 1 of the healthy siblings in family II). Early truncation of the extracellular domain of the protein is predicted as the result of both mutations. No mutation in CD3D was identified in PI-3. A homozygous 2-bp deletion at nucleotide 128 in exon 5 of CD3E was identified in this patient. This deletion leads to a frameshift at residue 43 and the generation of a nonsense codon located 13 residues downstream of this residue. $\mathrm{A} C \mathrm{CD} 3 \varepsilon$ chain truncated in its extracellular domain is predicted as the result of this deletion (Figure 2). This $C D 3 E$ mutation was found in combination with a wildtype allele in DNA from the father of the patient. DNA from the patient's mother was not available for this study. No similar mutations in the $C D 3 D$ and $C D 3 E$ genes were found from a combination of standard and single-base sequence analyses of more than 90 control chromosomes, which indicates that these nonsense mutations are not common polymorphisms. However, the mutation found in PIII-2 has been previously reported in a separate kindred (11).

Differentiation is blocked at the intermediate $C D 4^{+}$stage in $C D 3 \delta$-deficient thymocytes. Thymi from fetuses PIII-2 and PIII-3 were small. Their weight was one-third of that expected for a fetus of that gestational age ( $0.9 \mathrm{~g}$ for PIII- 2 compared with an expected value of $2.7 \pm 1.4 \mathrm{~g}$ at 24 weeks' gestation, and $0.3 \mathrm{~g}$ for PIII- 3 compared with an expected value of $0.9 \pm 0.3 \mathrm{~g}$ at 22 weeks' gestation). Results from the immunohistochemical analysis of the thymi from these affected fetuses were compared with those obtained from a control to assess in more detail the effects of defective $C D 3 D$ expression on $T$ cell development. The thymi of PIII-2 and PIII- 3 and the control showed a similar lobulated architecture. However, H\&E-stained sections of the thymic tissues revealed that corticomedullary differentiation was lacking in PIII-2 and PIII-3, whereas the control thymus displayed a distinct corticomedullary junction (Figure 3, A and B, and Figure 4). Thymic epithelial cells were present in PIII-2 and PIII-3 (Figure 3B), and only a very few Hassall corpuscles were observed (Figure 3B). Cytoplasmic $\mathrm{CD} 3 \varepsilon^{+}$thymocytes were abundant, although their density was reduced by $40 \%$ in comparison with that of normal thymus (Figures $3 \mathrm{C}$ and $4 \mathrm{~A})$. The majority of the $\mathrm{CD} 3 \varepsilon^{+}$thymocytes from the patients and the control did not express cKit and CD34, which are markers for early thymocytes (data not shown), but did express CD4 (Figure 3D). However, only a small subset of thymocytes $(<10 \%)$ expressed CD 45 RO and $\mathrm{CD} 8 \alpha$ (Figure 3, E and F) as compared with the thymocytes from control thymus. Compared with the cortical $\mathrm{CD} 3 \varepsilon^{+}$thymocytes from the control fetus, the fraction of cycling $\mathrm{CD} 3 \varepsilon^{+}$thymocytes detected by anti-Ki67 labeling was lower in PIII-3 (a 22-week-old fetus) and almost absent in PIII-2 (a 24-week-old fetus) (Figures 3G and 4B). Together, these results suggest that $\mathrm{T}$ cell development was blocked between the differentiation stage of the $\mathrm{CD}^{+}{ }^{+} \mathrm{CD} 4^{+} \mathrm{CD} 8{ }^{-} \mathrm{CD} 45 \mathrm{RO}^{-}$subset, 


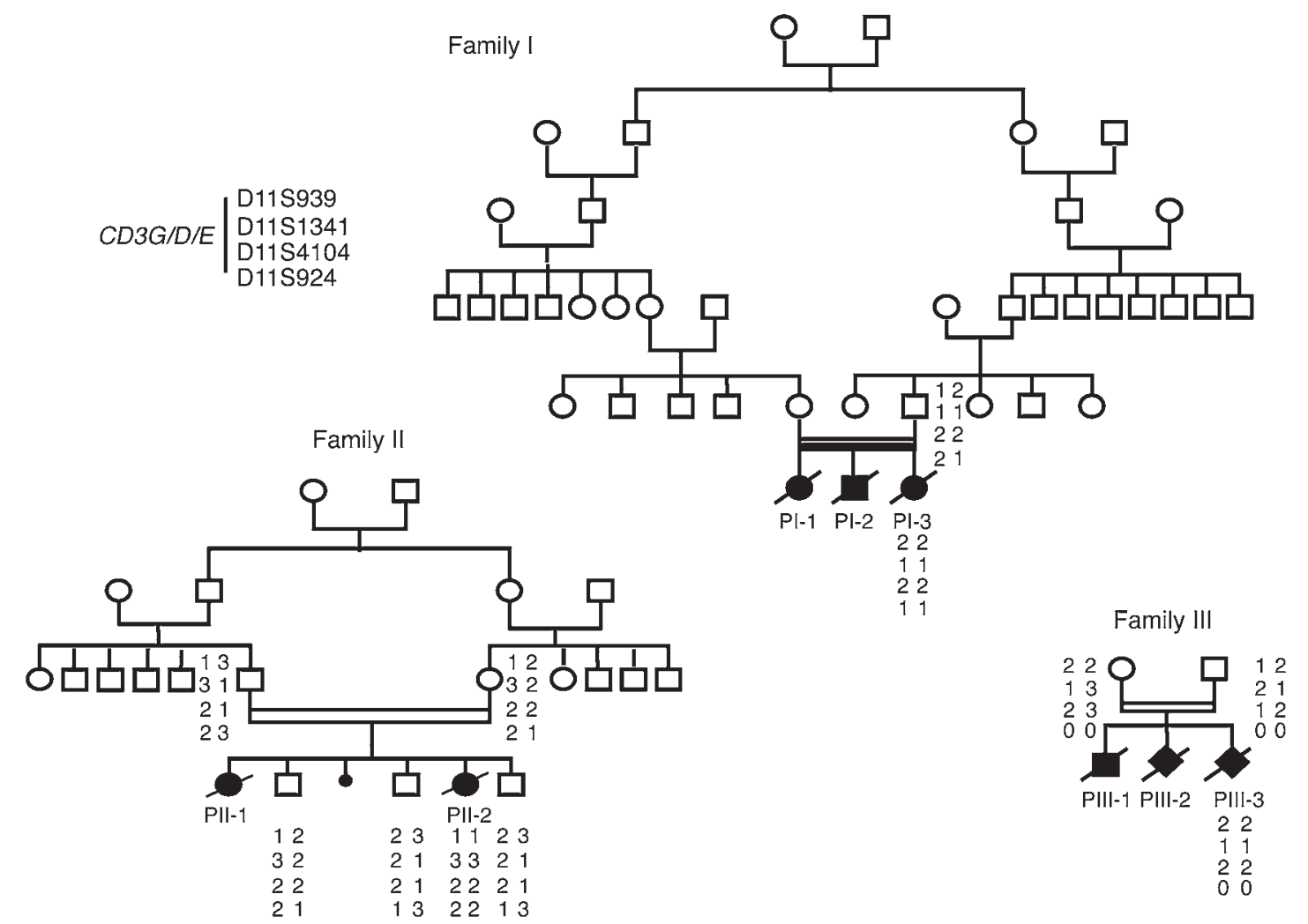

Figure 1

Pedigree and linkage analysis of the 3 families studied. Haplotype analysis of polymorphic markers spanning the CD3G/D/E locus on chromosome 11q23 revealed homozygous haplotype segregations in the patients studied. Polymorphic markers were ordered according to the National Center for Biotechnology Information Homo sapiens map (35). The phase in the patients was determined by analysis of parental genotype.

corresponding to intermediate single positive (ISP) thymocytes, and that of the $\mathrm{CD}^{+} \mathrm{CD} 4^{+} \mathrm{CD} 8{ }^{+} \mathrm{CD} 45 \mathrm{RO}^{+}$subset, corresponding to double positive thymocytes. Normal ISP thymocytes actively proliferate; therefore the low proliferation rate observed for the patients suggests that $\mathrm{CD} 3 \delta$-deficient ISP thymocytes fail to proliferate in response to signals emanating from the pre-TCR.

Absence of T cells in lymph nodes from CD38-deficient patients. As lymph nodes are difficult to detect in SCID patients (21), we investigated whether lymph nodes were detectable in the CD38-deficient fetuses and also whether mature lymphocytes were present. Lymph nodes were found in the peripancreatic area in both PIII- 2 and PIII-3. The lymph nodes in the 2 patient fetuses and the control fetus were of similar size and number ( $>3$ per section) in the peripancreatic area on histological section and contained similar numbers of $\mathrm{CD} 20^{+} \mathrm{B}$ cells. In contrast, $\mathrm{CD} 3 \varepsilon^{+} \mathrm{T}$ cells were absent from the lymph nodes of both patients, whereas they were numerous in the paracortical zone of the control lymph nodes (Figure 5).

\section{Discussion}

We showed that the homozygous mutations found in the $C D 3 D$ and $C D 3 E$ genes of the families studied (mutations in $C D 3 D$ for families II and III and CD3E for family I) were associated with the $\mathrm{T}^{-} \mathrm{B}^{+} \mathrm{NK}^{+} \mathrm{SCID}$ phenotype. The consanguinity, sex ratio, and absence of the phenotype in heterozygous carriers demonstrate autosomal recessive inheritance of the defects. Other potential genetic causes of SCIDs were excluded on the basis of the phenotype and after analysis of the segregation of the polymorphic markers surrounding the SCID-associated loci. Deficiencies in $\gamma \mathrm{c}$ and Jak-3 are usually associated with a $\mathrm{T}^{-} \mathrm{B}^{+} \mathrm{NK}^{-}$phenotype, and thus the possibility that the mutations lay within these genes was excluded. Similarly, an adenosine deaminase deficiency was exclud$\mathrm{ed}$, as this generally results in $\mathrm{T}^{-} \mathrm{B}^{-} \mathrm{NK}^{-}$SCID. An IL-7R $\alpha$ deficiency, which results in a $\mathrm{T}^{-} \mathrm{B}^{+} \mathrm{NK}^{+}$phenotype (8) identical to that observed in the 3 families, could be ruled out by genetic analysis.

Both CD38- and CD3e-deficient patients had complete deficiencies in peripheral $\mathrm{T}$ cells, including $\gamma / \delta \mathrm{T}$ cells. Both the

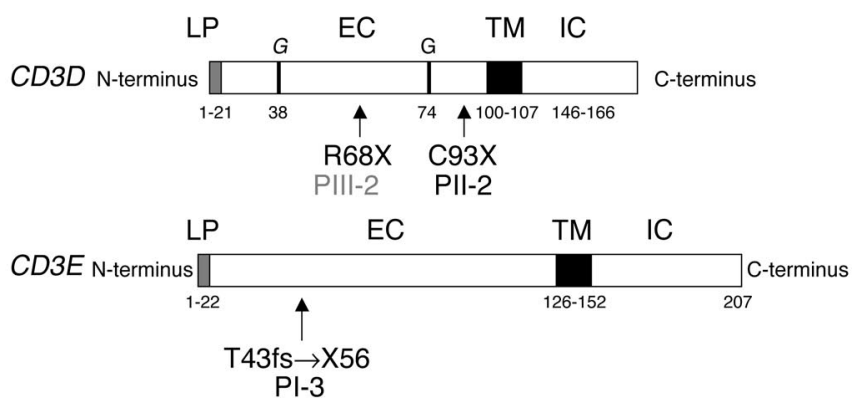

Figure 2

Schematic representation of the $C D 3 D$ and $C D 3 E$ gene mutations. The extracellular (EC), transmembrane (TM), and intracellular (IC) regions, the leader peptide (LP), and the glycosylation sites (G) are shown. $X$, stop codon; fs, frameshift. 


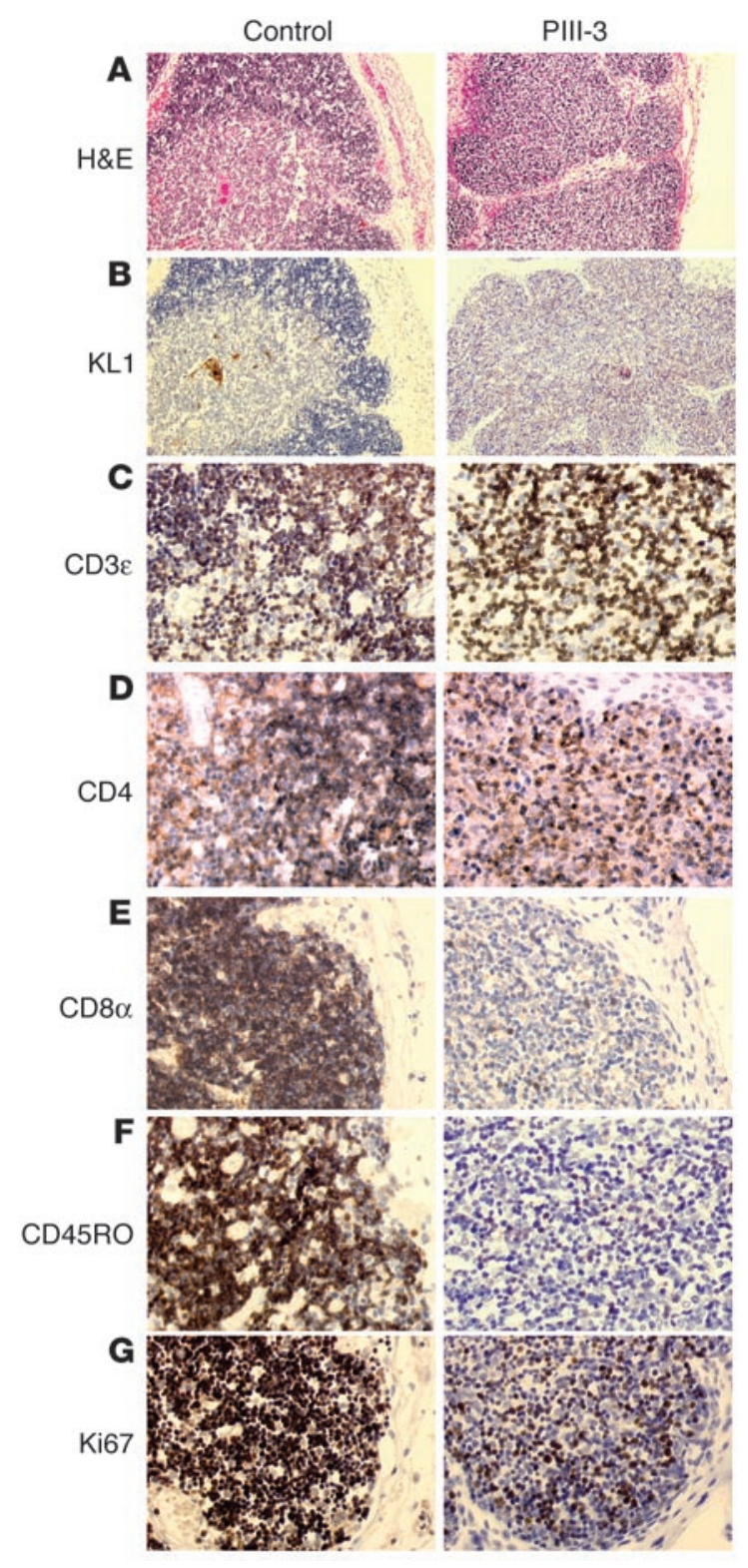

$C D 3 D$ gene mutations resulted in a premature stop codon, and, as the truncated products do not encompass the transmembrane domain, they were not predicted to be expressed. It is important to note that the R68X mutation was also found in 3 patients from an unrelated kindred with an identical SCID phenotype (11). In contrast to our study, where T cells were absent from the periphery, the only CD3E deficiency described in a previous report did not lead to impaired $\mathrm{T}$ cell development (22). This discrepancy between the phenotypes is probably related to differences in the extent of the CD3E deficiency. In the previous study, residual $\mathrm{CD} 3 \varepsilon$ expression was found at the $T$ cell surface as a consequence of a splice-site mutation on 1 allele that did not totally abrogate the normal intron 7 splicing (22). In contrast, the homozygous CD3E mutation in family I in our study created a premature stop codon near the start of the extracellular domain and thus resulted in the absence of $C D 3 \varepsilon$. These observations show that an absence of $\mathrm{CD} 3 \varepsilon$ or $\mathrm{CD} 3 \delta$ completely abrogates human $\mathrm{T}$ cell

\section{Figure 3}

Immunohistology of the thymus from PIII-3 (a 22-week-old fetus) and a control fetus. Sections from the PIII-3 and control thymus were stained with $\mathrm{H} \& \mathrm{E}(\mathbf{A})$, and with antibodies to cytokeratin $(\mathrm{KL} 1)(\mathbf{B}), \mathrm{CD} \varepsilon \varepsilon(\mathbf{C})$, CD4 (D), CD8 $\alpha(E)$, CD45RO (F), and Ki67 (G). Similar results were obtained for both PIII-3 and PIII-2, with the exception that the Ki67 antibody labeled more cells in PIII-3 than in PIII-2 (a 24-week-old fetus) (see Figure 4). Magnification, $\times 40$.

development. The assumption of the role of $\mathrm{CD} 3 \varepsilon$ also requires confirmation by description of additional cases.

It was not possible to assess at what stage the $\mathrm{CD} 3 \varepsilon$ deficiency blocked $\mathrm{T}$ cell differentiation in patients from family $\mathrm{I}$, as no thymus material was available. In the mouse, $C d 3 \varepsilon$ gene inactivation results in a complete block at the $\mathrm{CD} 44^{-} \mathrm{CD} 25^{+}$double negative $\mathrm{T}$ cell differentiation stage, showing that $\mathrm{CD} 3 \varepsilon$ is essential for transduction of the PreT $\alpha /$ TCR $\beta$ receptor survival, proliferation, and progression signals $(23,24)$. It is possible that the $\mathrm{T}$ cell differentiation is disrupted at the same stage in the CD3E-deficient human thymus.

In contrast, the thymi of patients or fetuses with the CD38 deficiency were analyzed both in this study and in that of Dadi et al. (11). Dadi et al. reported that $\mathrm{T}$ lymphocyte development was blocked at the pre-TCR expression stage (11), since the levels of CD 4 and $C D 8 \alpha / \beta$ mRNAs and proteins in thymocytes from the patients were lower than those in the thymocytes of the controls (11). By performing an immunohistochemical analysis, we found that $\mathrm{T}$ cell differentiation was blocked slightly later in our patients, at the ISP stage. Most cells did not express cKit or CD34, and the majority of $\mathrm{T}$ cells were $\mathrm{CD} 4^{+}$ and $\mathrm{CD}^{2} 4 \mathrm{RO}^{-} \mathrm{CD} 8^{-}$, both of which are characteristic of ISP cells (25). In mice and humans, ISP cells actively proliferate, a process that is driven by signals emanating from the pre-TCR and that results in the generation of double positive thymocytes. The finding that cell proliferation, as revealed by anti-Ki67 labeling, was strongly impaired in the patients in our study suggests that the defect in the pre-TCR-mediated proliferation is at the ISP stage.

The differences in thymic phenotypes between the 2 studies are not highly significant, since the way the thymus samples were analyzed differed and the thymi used by Dadi et al. (11) originated from infants rather than a fetus. It is possible that in the absence of CD $3 \delta$, some PreT $\alpha^{+} / \mathrm{TCR} \beta^{+}$thymocytes survive and progress up to the stage of ISP cells. Even if this is the case, the results from the 2 studies show that $\mathrm{CD} 3 \delta$ is required during the early stage of human thymopoiesis, most likely as a mediator of the signals transmitted from the PreT $\alpha / \mathrm{TCR} \beta$ receptor. In addition, TCR $\gamma / \delta^{+} \mathrm{T}$ cells were not detected in patients with CD3 $\delta$ deficiency (11), which demonstrates that $\mathrm{CD} 3 \delta$ is also required for the development of these $\mathrm{T}$ cells. This phenotype differs from that observed in the $\mathrm{CD}^{-/-} \mathrm{\delta}^{-/}$murine model (18). The size of the peripheral T cell pool in these mice is $10 \%$ of that in the controls, and the $\mathrm{T}$ cells express a reduced number of membrane TCR $/ \gamma \varepsilon \xi \mathrm{CD} 3$ complexes; these findings indicate that the $\mathrm{CD} 3 \delta$ deficiency blocks $\mathrm{T}$ cell development at the $\mathrm{CD} 4^{+} \mathrm{CD} 8^{+}$ stage in mice. This interspecies difference could be the consequence of CD3 $\delta$ playing a different role in CD3-pre-TCR or CD3-TCR complex assembly or signaling in humans versus mice. CD3 8 is involved in the ERK activation associated with positive selection in mice (26). Therefore, it is presumed that $\mathrm{CD} 3 \delta$ plays an additive role at an earlier stage of $\mathrm{T}$ cell differentiation in mice than in humans.

The phenotype of the CD38- and CD3e-deficient patients sharply contrasts with that associated with CD3 $\gamma$ deficiency $(19,27)$. In $\mathrm{CD} 3 \gamma$-deficient individuals, $\mathrm{T}$ cell differentiation is not impaired 

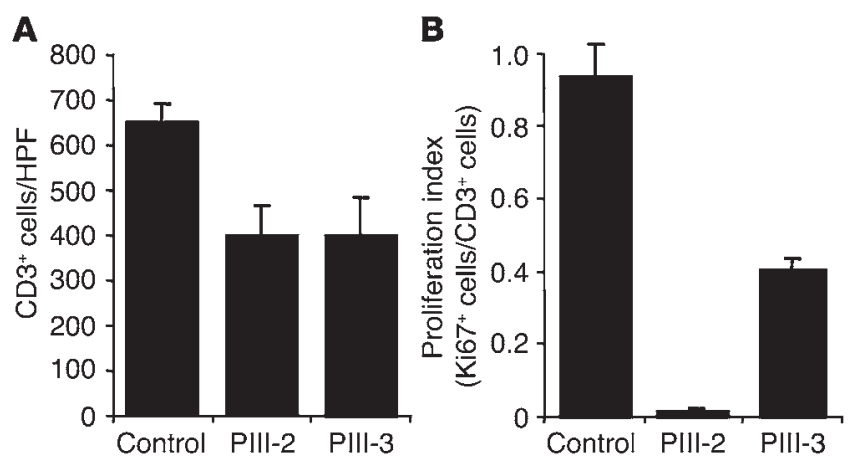

\section{Figure 4}

$\mathrm{CD}^{+}$thymocyte counts and their proliferation in the thymi from PIII-2 and PIII-3 compared with the thymus from the control fetus. (A) Number of $\mathrm{CD}^{+}$thymocytes per high-power field (HPF; magnification, $\times 40$ ). Fields were chosen at random from CD3 $\varepsilon$ antibody-labeled sections of PIII-3, PIII-2, and control thymi. CD3 antibody-labeled cells were counted on photographs of 3 fields per sample. The results are presented as the mean and SD of the 3 measurements. (B) Percentage of Ki67+ thymocytes among the $\mathrm{CD}^{+}$thymocytes. Fields were photographed at random from Ki67 antibody-labeled sections of PIII-3, PIII-2, and control thymus (magnification, $\times 40$ ). Ki67 antibody-labeled cells were then counted in 3 fields per sample. The results were plotted against the values obtained for CD3.

and mature CD4 T cells express the $(\delta \varepsilon)_{2} \zeta_{2}$ (28). These observations emphasize the facts that the CD3 subunits fulfill different functions during TCR-CD3 complex assembly and signaling, and that the more closely related $\delta$ and $\gamma \mathrm{CD} 3$ subunits are not interchangeable.

We have identified a novel genetic cause of SCID, and, as a result, 10 mechanisms underlying this syndrome have now been characterized: ADA (3), $\gamma_{c}$ (6), Jak-3 (7), IL-7R $\alpha$ (8), Rag-1, Rag-2 (4), Artemis

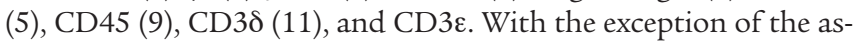
yet uncharacterized reticular dysgenesis form of SCID (29) and a few cases of $\mathrm{T}^{-} \mathrm{B}^{+} \mathrm{NK}^{+} \mathrm{SCID}$, these 10 deficiencies account for most cases of SCID. Therefore accurate molecular diagnosis and genetic counseling as well as potentially further treatments based on gene therapy can now be offered to the majority of SCID patients.

\section{Methods}

Patients. In family I, the parents were third cousins (Figure 1). Their daughter (patient I-1) died at 5 months of age of pneumonitis in 1983. No investigations were performed. In 1984, their second child (patient I-2) presented with diarrhea, pneumonitis, and oral candidiasis at 1 month of age. Lymphopenia $(1,105$ lymphocytes $/ \mu \mathrm{l})$ was detected, but no further immunological investigations were performed. This child died at 3 months of age. A postmortem tissue examination revealed a disseminated CMV infection. The adenosine deaminase level in the erythrocytes was normal. Because of the family history, patient I-3 was referred, at birth (1987), to Necker Hospital for an immunological evaluation (Table 1). A diagnosis of $\mathrm{T}^{-} \mathrm{B}^{+} \mathrm{NK}^{+} \mathrm{SCID}$ was made, and the patient received a haploidentical bone marrow transplant from her father. A disseminated adenovirus infection led to her death 25 days after bone marrow transplantation (BMT). In family II (Figure 1), the parents were first cousins. In 1987, their daughter (patient II-1) had diarrhea and failed to thrive at 3 months of age. At 5 months of age, she developed CMV hepatitis and died shortly afterward. Because of this family history, a second child (patient II-2) was referred at 6 days old for immunological investigations in 1997. The child was diagnosed with $\mathrm{T}^{-} \mathrm{B}^{+} \mathrm{NK}^{+}$SCID (Table 1). The patient received an HLA-haploidentical bone marrow transplant from her father but died 6 months after BMT from a disseminated Aspergillus infection. In family III, patient III-1 was born from related parents in 1992. At 2 months of age, he presented with oral and perineal candidiasis. He later developed protracted diarrhea and pneumonitis. At 5 months of age, immunological investigations led to a diagnosis of $\mathrm{T}^{-} \mathrm{B}^{+} \mathrm{SCID}$ (Table 1). He received a haploidentical bone marrow transplant from his mother but died from EBV infection 30 days after BMT. A prenatal diagnosis was made in family III during 2 subsequent pregnancies of the mother of the propositus (in 1997 and 1998, respectively). Tests were performed using immunofluorescence methods on fetal blood samples drawn at 23 and 21 weeks' gestation for the second and third pregnancies, respectively. No T cells were detected. Parents opted for termination of the pregnancy in both cases.

Immunological investigations. The following mAb's were used in the immunofluorescence studies: anti-CD3: Leu 4 (IgG2a; BD) and OKT3 (IgG1; OrthoPharmaceutical); anti-CD2: Leu 5b (IgG2a; BD); anti-CD4: Leu 3a (IgG1; BD); anti-CD8: Leu 2a (IgG1; BD); anti-CD56: Leu 19 (IgG1; BD); anti-CD19: J4 119 (IgG1; Immunotech); and anti-HLA DR: L243 (IgG2a; BD). Fluorescence staining was performed using PE- or FITC-conjugated $\mathrm{mAb}$ 's or by indirect immunofluorescence using a FITC-conjugated goat anti-mouse Ig (Nordic Immunological Laboratories). Cells were analyzed with a FACScan analyzer (BD). In PII-1, B cells were detected through expression of HLA class II. Serum Ig levels were determined by nephelometry. Informed consent for the study was obtained from the families in accordance with the Declaration of Helsinki. This study was approved by the INSERM Institutional Review Board.

Genotype analysis and mutation detection. Genomic DNA was extracted from peripheral blood cells (30), and genotype analysis was performed as previously described (31) using 19p13.1 and 5p13 chromosome markers, which spanned the JAK3 and $I L 7 R$ loci, respectively $(32,33)$, and markers neighboring the $C D 3 G / D / E$ locus on chromosome 11q23 (D11S939, D11S1341, D11S4104, D11S924) (34).

Genomic and mRNA sequences of CD3D and CD3E (accession numbers NT033899, NM000732, and NM000733) were obtained from the National Center for Biotechnology Information (35). CD3D and CD3E exons were amplified from genomic DNA by PCR and sequenced in both directions. PCR was carried out using the BigDye Terminator Cycle Sequencing Ready Reaction Kit (Applied Biosystems) and standard methods. The sequence of primers used is as follows: CD3E.ex1:5'-CGCCATCTTAGTAAAGTAAC-3'; CD3Eex1r 5'-CTGGGTAAGTTGGAATG-3'; CD3E.ex 2f:5'-GAACCCCTGGTCAATAC3'; CD3E.ex 2r: 5'-GGTAGATGGCAGAAACTC-3'; CD3E.ex 3f: 5'-GATGTAAGGCCCATAACA-3'; CD3E.ex 3r: 5'-AGGCAAGTTTCATACGG-3'; CD3E. ex 4f: 5'-CAAGACTCGGGGTTCCTA-3'; CD3E.ex 4r: 5'-CAGGGACATGGGGACTT-3'; CD3E.ex 5f: 5'-GTCACTAATTTGCCTTTT-3'; CD3E.ex

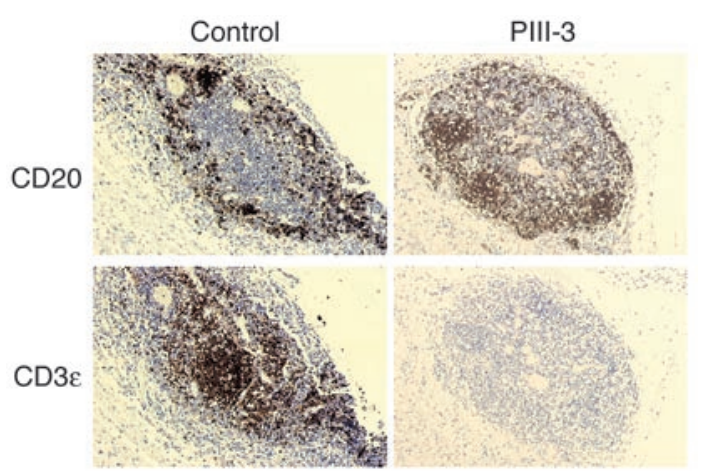

\section{Figure 5}

Immunohistology of a lymph node from the PIII-2 (24-week-old) fetus and a control (24-week-old) fetus. Peripancreatic lymph node sections from PIII-3, PIII-2, and control thymi were labeled with anti-CD20 mAb and anti-CD3 $\varepsilon$ mAb. Magnification, $\times 40$. 
5r:5'-CACAGACTGGGGTTAC-3'; CD3E.ex 6f: 5'-GTGGCAAGCGTGTGAGT3'; CD3E.ex 6r: 5'-GGAAGGAGGGGAGTGATG-3'; CD3E.ex 7f: 5'-GAGCGGTAGAGGAGAGAACA-3'; CD3E.ex7r: 5'-GTGGCACATAGAGGGTTGT-3'; CD3E.ex 8f: 5'-CTCCCGCACCACTGACC-3'; CD3E.ex 8r: 5'-GGGATGGGGGACAACTTACA-3'; CD3Dex1f: 5'-CAACGGCAGAAAGCAGAGA3'; CD3Dex1r: 5'-GAAATCCCGGGCTCAAGT-3'; CD3Dex2f: 5'-GGTGGGGAACTTTCTGAAG-3'; CD3Dex2r: 5'-CATGACCCCTACTGCTCTGT-3'; CD3Dex 3f: 5'-CTTGTTCAAGGTCCCAAAAG-3'; CD3Dex3r: 5'-ACCACCAGCCCCATTC-3'; CD3Dex 4+5f:5'-ATCTGCTAGGCCATTGATGT-3'; CD3Dex4+5r: 5'-CCAAGCAAGTCAGGACAAC-3'.

Immunopathology of thymus and lymph nodes. Tissues from fetuses PIII-2 (24 weeks old) and PIII-3 (22 weeks old) and a non-immunodeficient 24-weekold control fetus were obtained at autopsy after informed consent of the families. Five-micrometer serial sections of formalin-fixed paraffin-embedded tissues were mounted on SuperFrost Plus glass slides (Consortium du Matériel pour Laboratoire), air-dried, deparaffinized, and stained with $\mathrm{H} \& \mathrm{E}$ or with the antibodies listed below followed by a goat anti-mouse peroxidaseconjugated antibody (DakoCytomation S.A.S.). Diaminobenzidine (DAB; DakoCytomation S.A.S.) was used as substrate for peroxidase (36). The mAb's used were anti-Ki67 (MiB1, mouse IgG1), anti-cytokeratin (KL1, mouse IgG1), anti-CD34 (QBEND10, mouse IgG1; Immunotech), anti-CD4 (1F6, mouse IgG1; BD), anti-CD8 (C8/144B, IgG1), anti-CD45RO (UCHL1, IgG2a), anti-CD20 (L26, mouse IgG2a), anti-CD3ع (rabbit polyclonal antibody), and anti-cKit (rabbit polyclonal antibody; DakoCytomation S.A.S.).

\section{Acknowledgments}

We thank P. Lutz (Service d'Hématologie Pédiatrique, Hôpitaux Universitaires de Strasbourg) for providing the clinical care for the patients, and B. Gasser (Département d'Anatomopathologie, Hôpitaux Universitaires de Strasbourg) for donating fetuses. We thank Nathalie Lambert, Cécile Dumont (Centre d'Étude des Déficits Immunitaires), and Monique Perennec (Laboratoire d'Anatomie Pathologique) for expert technical assistance, and Sophie Bécavin for typing the manuscript. This work was supported by an institutional INSERM grant.

Received for publication June 30, 2004, and accepted in revised form August 24, 2004.

Address correspondence to: Françoise Le Deist, Centre d'Étude des Déficits Immunitaires, INSERM U 429, Hôpital Necker-Enfants Maladies, 149 rue de Sèvres, 75743 Paris Cedex 15, France. Phone: 33-1-44-49-50-88; Fax: 33-1-42-73-06-40; E-mail: francoise. ledeist@nck.ap-hop-paris.fr.

Claire Soudais's present address is: Laboratoire de Génétique Humaine des Maladies Infectieuses, INSERM U 550, Faculté Necker, Paris, France.
1. Chapel, H., Geha, R., and Rosen, F. 2003. Primary immunodeficiency diseases: an update. Clin. Exp. Immunol. 132:9-15.

2. Buckley, R.H. 2004. Molecular defects in human severe combined immunodeficiency and approaches to immune reconstitution. Annu. Rev. Immunol. 22:625-655.

3. Hirschhorn, R. 1999. Immunodeficiency disease due to deficiency of adenosine deaminase. In Primary immunodeficiency diseases. A molecular and genetic approach. H.D. Ochs, C.I.E. Smith, and J.M. Puck, editors. Oxford University Press. New York, New York, USA. 121-139.

4. Schwarz, K., et al. 1996. RAG mutations in human B cell-negative SCID. Science. 274:97-99.

5. Moshous, D., et al. 2001. Artemis, a novel DNA double-strand break repair/V(D)J recombination protein, is mutated in human severe combined immune deficiency. Cell. 105:177-186.

6. Leonard, W.J., Noguchi, M., Russell, S.M., and McBride, O.W. 1994. The molecular basis of Xlinked severe combined immunodeficiency: the role of the interleukin-2 receptor gamma chain as a common gamma chain, gamma c. Immunol. Rev. 138:61-86.

7. Macchi, P., et al. 1995. Mutations of Jak-3 gene in patients with autosomal severe combined immune deficiency (SCID). Nature. 377:65-68.

8. Puel, A., Ziegler, S.F., Buckley, R.H., and Leonard, W.J. 1998. Defective IL7R expression in $\mathrm{T}(-) \mathrm{B}(+) \mathrm{NK}(+)$ severe combined immunodeficiency. Nat. Genet. 20:394-397.

9. Kung, C., et al. 2000. Mutations in the tyrosine phosphatase CD45 gene in a child with severe combined immunodeficiency disease. Nat. Med. 6:343-345

10. Tchilian, E.Z., et al. 2001. A deletion in the gene encoding the CD45 antigen in a patient with SCID. J. Immunol. 166:1308-1313.

11. Dadi, H.K., Simon, A.J., and Roifman, C.M. 2003. Effect of CD3 $\delta$ deficiency on maturation of alpha/ beta and gamma/delta T-cell lineages in severe combined immunodeficiency. N. Engl. J. Med. 349:1821-1828.

12. Spits, H. 2002. Development of alphabeta T cells in the human thymus. Nat. Rev. Immunol. 2:760-772.
13. Anderson, G., Harman, B.C., Hare, K.J., and Jenkinson, E.J. 2000. Microenvironmental regulation of $\mathrm{T}$ cell development in the thymus. Semin. Immunol. 12:457-464.

14. Blackburn, C.C., and Manley, N.R. 2004. Developing a new paradigm for thymus organogenesis. Nat. Rev. Immunol. 4:278-289.

15. Sun, Z.J., Kim, K.S., Wagner, G., and Reinherz, E.L. 2001. Mechanisms contributing to T cell receptor signaling and assembly revealed by the solution structure of an ectodomain fragment of the CD3 epsilon gamma heterodimer. Cell. 105:913-923.

16. Borroto, A., Mallabiabarrena, A., Albar, J.P., Martinez, A.C., and Alarcon, B. 1998. Characterization of the region involved in $\mathrm{CD} 3$ pairwise interactions within the T cell receptor complex. J. Biol. Chem. 273:12807-12816.

17. Haks, M.C., Krimpenfort, P., Borst, J., and Kruisbeek, A.M. 1998. The CD3gamma chain is essential for development of both the TCRalphabeta and TCRgammadelta lineages. EMBO J. 17:1871-1882.

18. Dave, V.P., et al. 1997. CD3 delta deficiency arrests development of the alpha beta but not the gamma delta T cell lineage. EMBOJ. 16:1360-1370.

19. Arnaiz-Villena, A., et al. 1993. T lymphocyte signalling defects and immunodeficiency due to the lack of CD3 gamma. Immunodeficiency. 4:121-129.

20. Zapata, D.A., et al. 1999. Conformational and biochemical differences in the TCR.CD3 complex of CD8(+) versus CD4(+) mature lymphocytes revealed in the absence of CD3gamma. J. Biol. Chem. 274:35119-35128.

21. Gossage, D.L., and Buckley, R.H. 1990. Prevalence of lymphocytopenia in severe combined immunodeficiency. N. Engl. J. Med. 323:1422-1423.

22. Soudais, C., de Villartay, J.P., Le Deist, F., Fischer, A., and Lisowska-Grospierre, B. 1993. Independent mutations of the human CD3- $\varepsilon$ gene resulting in a $\mathrm{T}$ cell receptor/CD3 complex immunodeficiency. Nat. Genet. 3:77-81.

23. Sommers, C.L., et al. 2000. Function of CD3 $\varepsilon$-mediated signals in T cell development. J. Exp. Med. 192:913-919.

24. Wang, N., et al. 1998. Expression of a CD3 epsilon transgene in CD3 epsilon(null) mice does not restore CD3 gamma and delta expression but efficiently rescues $\mathrm{T}$ cell development from a subpopulation of prothymocytes. Int. Immunol. 10:1777-1788.

25. Fukuhara, K., et al. 2002. A study on CD45 isoform expression during T-cell development and selection events in the human thymus. Hum. Immunol. 63:394-404.

26. Delgado, P., Fernandez, E., Dave, V., Kappes, D., and Alarcon, B. 2000. CD38 couples T-cell receptor signalling to ERK activation and thymocyte positive selection. Nature. 406:426-430.

27. Timon, M., et al. 1993. Selective disbalances of peripheral blood T lymphocyte subsets in human CD3 gamma deficiency. Eur.J. Immunol. 23:1440-1444.

28. Zapata, D.A., et al. 2004. Biochemical differences in the alphabeta $T$ cell receptor. $C D 3$ surface complex between CD8+ and CD4+ human mature T lymphocytes. J. Biol. Chem. 279:24485-24492.

29. Roper, M., Parmley, R.T., Crist, W.M., Kelly, D.R., and Cooper, M.D. 1985. Severe congenital leukopenia (reticular dysgenesis). Immunologic and morphologic characterizations of leukocytes. Am.J. Dis. Child. 139:832-835.

30. Barrat, F.J., et al. 1996. Genetic and physical mapping of the Chediak-Higashi syndrome on chromosome 1q42-43. Am. J. Hum. Genet. 59:625-632.

31. Pastural, E., et al. 2000. Two genes are responsible for Griscelli syndrome at the same $15 \mathrm{q} 21$ locus. Genomics. 63:299-306.

32. Hoffman, S.M., et al. 1997. JAK3 maps to human chromosome $19 \mathrm{p} 12$ within a cluster of protooncogenes and transcription factors. Genomics. 43:109-111.

33. Lynch, M., Baker, E., Park, L.S., Sutherland, G.R., and Goodwin, R.G. 1992. The interleukin-7 receptor gene is at 5p13. Hum. Genet. 89:566-568.

34. Dib, C., et al. 1996. A comprehensive genetic map of the human genome based on 5,264 microsatellites. Nature. 380:152-154.

35. National Center for Biotechnology Information Map viewer. http://www.ncbi.nlm.nih.gov/mapview.

36. Cordell, J.L., et al. 1984. Immunoenzymatic labeling of monoclonal antibodies using immune complexes of alkaline phosphatase and monoclonal anti-alkaline phosphatase (APAAP complexes). J. Histochem. Cytochem. 32:219-229. 